\title{
Mechanisms of spikelet generation in cortical pyramidal neurons
}

\author{
Martina Michalikova ${ }^{1 *}$, Michiel Remme $^{1}$, Richard Kempter ${ }^{1,2}$ \\ From 24th Annual Computational Neuroscience Meeting: CNS*2015 \\ Prague, Czech Republic. 18-23 July 2015
}

Spikelets are brief, spike-like depolarizations of small amplitude $(<20 \mathrm{mV})$ that can be measured in somatic intracellular recordings. Prominent spikelet activity was demonstrated in hippocampal CA1 pyramidal neurons in awake behaving [1,2] and anesthetized animals [3]. However, spikelets are rarely observed in vitro, and basic mechanisms underlying their generation in pyramidal neurons are not well understood.

Here we investigate the emergence of spikelets using mathematical analysis and numerical simulations of compartmental single-neuron models. Somatic spikelets are produced in the models upon orthodromic (somatic) stimulation. We find that spikelet occurrence depends on three main factors: A) Activation voltages of somatic channels need to be larger by several millivolts $(\sim 10 \mathrm{mV})$ than activation voltages of axonal sodium channels. B) The spike initiation zone (axon initial segment, AIS) has to be electrically segregated from the soma. C) The impedance mismatch between soma and AIS needs to be sufficiently large. In this way, weak orthodromic stimuli can trigger APs at the AIS that fail to activate somatic sodium channels and manifest as somatic spikelets. Stronger stimuli lead to full-size APs at the soma, either through axonal APs that backpropagate to the soma ('shouldered APs') or direct somatic AP generation ('full-blown APs').

Through analysis and simulations we isolated the cell parameters that allow for spikelet generation and identified possible causes of spikelet absence in in vitro preparations: First, the dendritic current sink in vitro is diminished due to "dendritic pruning" in slices. Next, the fraction of sodium channels usually available for (somatic) spiking is larger in vitro due to the overall lower spiking activity and lower resting membrane

\footnotetext{
* Correspondence: martina.michalikova@hu-berlin.de

${ }^{1}$ Institute for Theoretical Biology, Department of Biology, Humboldt-

Universität zu Berlin, 10115 Berlin, Germany

Full list of author information is available at the end of the article
}

potential. Finally, the difference in activation voltages between somatic and axonal sodium channels under in vitro conditions might be smaller than under in vivo conditions as the activation voltage of sodium channels might be controlled by neuronal activity, which is typically much higher in vivo than in vitro.

\section{Conclusions}

In our models, somatic spikelets represent APs that are only propagated down the axon, but are not backpropagated to the soma and the dendrites. Consequently, such a mechanism might be involved in the control of dendritic plasticity and/or in the homeostatic regulation of somato-dendritic firing rates without affecting the axonal output of a neuron.

\section{Acknowledgements}

This work was supported by the Einstein Foundation Berlin and the German Federal Ministry of Education and Research (01GQ0901, 01GQ1001A, 01GQ0972).

\section{Authors' details}

${ }^{1}$ Institute for Theoretical Biology, Department of Biology, HumboldtUniversität zu Berlin, 10115 Berlin, Germany. ${ }^{2}$ Bernstein Center for Computational Neuroscience, 10115 Berlin, Germany.

Published: 18 December 2015

\section{References}

1. Epsztein J, Lee AK, Chorev E, Brecht M: Impact of spikelets on hippocampal CA1 pyramidal cell activity during spatial exploration. Science 2010, 327(5964):474-477.

2. Harvey CD, Collman F, Dombeck DA, Tank DW: Intracellular dynamics of hippocampal place cells during virtual navigation. Nature 2009, 461(7266):941-946.

3. Chorev E, Brecht M: In vivo dual intra- and extracellular recordings suggest bidirectional coupling between CA1 pyramidal neurons. J Neurosci 2013, 33(11):4815-4824.

doi:10.1186/1471-2202-16-S1-P121

Cite this article as: Michalikova et al:: Mechanisms of spikelet generation in cortical pyramidal neurons. BMC Neuroscience 2015 16(Suppl 1):P121. 